\title{
A homeopatia no SUS na perspectiva de estudantes da área da saúde
}

\author{
Homeopathy in the Single Health System from \\ the Perspective of Students in the Health \\ Professions Gecioni Loch-Neckel
}

\author{
Gecioni Loch-Neckel ${ }^{\mathrm{I}}$ \\ Françoise Carmignan ${ }^{\text {II }}$ \\ Maria Aparecida Crepaldi ${ }^{\mathrm{I}}$
}

\section{PALAVRAS-CHAVE}

- Homeopatia

- Sistema Único de Saúde

- Estudantes de Ciências da Saúde

- Ciências da Saúde

- Representações Sociais.

\section{KEY WORDS}

- Homeopathy

- Single Health System

- Students, Health Occupations

- Health Science

- Social Representations
Recebido em: 22/11/2008 Reencaminhado em: 28/01/2009 Aprovado em: 02/07/2009

\begin{abstract}
R E S U M O
A homeopatia foi reconhecida como especialidade médica em 1980. Após a criação do SUS, alguns estados e municípios brasileiros começaram a oferecer atendimento homeopático aos usuários dos serviços públicos de saúde. Em 2006, foi editada a Portaria 971, que assegura o acesso à homeopatia a estes usuários, entre outras práticas integrativas e complementares. Diante disto, investigamos as percepções de estudantes dos cursos de Farmácia, Medicina e Odontologia sobre a homeopatia e sua prática no SUS, tendo por fundamento a teoria das representações sociais. Estas representações foram construídas com os seguintes parâmetros: a homeopatia como terapêutica; a relação entre a homeopatia, o SUS e o princípio da integralidade. Os estudantes souberam correlacionar o atendimento integral ao sujeito à compreensão de suas necessidades a partir de sua realidade. Constatou-se desconhecimento sobre os pressupostos teóricos da homeopatia e o não reconhecimento da incorporação da homeopatia no SUS, para a maioria dos estudantes. Pode ser sugerido que a inserção dos futuros profissionais no contexto das atividades em saúde e o acesso a outras racionalidades permitiriam uma escolha mais lúcida de suas práticas profissionais.
\end{abstract}

\section{A B S T R A C T}

In Brazil, homeopathy was officially recognized as a medical specialty by the National Medical Board in 1980. Since the creation of the Unified National Health System (SUS), some Brazilian States and municipalities have begun to offer homeopathic care to users of public health services. In 2006, the government issued Ruling 971, which guarantees access to homeopathic care for these users, in addition to other integrative and complementary therapies. Based on the above, we studied the perceptions of undergraduate students of Pharmacy, Medicine, and Dentistry concerning homeopathy and its practice in the National Health System, based on the theory of social representations. These representations were constructed with the following parameters: homeopathy as therapy; and the relationship between homeopathy, the National Health System, and the principle of comprehensiveness. Students were able to correlate comprehensive patient care with understanding patients' needs based on their reality. Most students lacked knowledge on the theoretical premises of homeopathy and were unaware that it had been incorporated into the National Health System. The findings suggest that the inclusion of future professionals in the context of health activities and access to other rationales would allow a clearer choice of their professional practices.

\footnotetext{
I Universidade Federal de Santa Catarina, Florianópolis, SC, Brasil.

${ }^{I I}$ Universidade da Região de Joinville, Joinville, SC, Brasil.
} 


\section{INTRODUÇÃO}

A homeopatia é um sistema terapêutico de caráter sistêmico, fundamentado no princípio vitalista e na lei dos semelhantes, postulada por Hipócrates no século IV a.C. Sua prática terapêutica consiste em curar os doentes valendo-se de remédios preparados em diluições infinitesimais e capazes de produzir no homem aparentemente sadio sintomas semelhantes aos da doença que devem curar num paciente específico. É uma terapêutica médica focada na compreensão do indivíduo dentro do seu contexto e no aspecto pessoal de suas reações diante das agressões. Na aplicação terapêutica desses pressupostos, valoriza-se a individualidade humana, elegendo, dentre os milhares de substâncias experimentadas, aquela que apresente a "totalidade de sintomas característicos de cada paciente" (nos aspectos psíquicos, emocionais, gerais e clínicos). Para um mesmo tipo de doença, são empregados medicamentos distintos para cada indivíduo enfermo, uma vez que o processo diagnóstico é centrado no enfermo - e não na doença ${ }^{1,2}$.

A homeopatia foi desenvolvida e difundida por Samuel Hahnemann no século 18, após extensos estudos e reflexões baseados na observação clínica e em experimentos realizados na época. Hahnemann sistematizou os princípios filosóficos e doutrinários da homeopatia em suas obras Organon da Arte de Curar e Doenças Crônicas. Desde então, ocorreu grande expansão da homeopatia por várias regiões do mundo, e hoje ela está firmemente implantada em diversos países da Europa, das Américas e da Ásia. No Brasil, a homeopatia foi introduzida por Benoit Mure em 1840, tornando-se rapidamente uma nova opção de tratamento para a população ${ }^{3}$.

A reforma sanitária brasileira e a criação do Sistema Único de Saúde (SUS), na década de 1980, se mostraram ressonantes aos princípios doutrinários homeopáticos, abrindo as portas à entrada da homeopatia no SUS. Este foi regulamentado pelas Leis 8.080 e 8.142 de 1990, e compreende princípios doutrinários como a equidade, a universalidade, a integralidade e princípios organizativos como hierarquização, descentralização, resolutividade e controle social ${ }^{4}$.

Afinando-se com os princípios básicos do SUS, alicerçada no controle social, a homeopatia busca consolidar como parâmetros da qualidade de sua prática:

- a integralidade: compreensão do sujeito enquanto unidade hierarquizada e indivisível, não sujeito à limitação de recortes patológicos em detrimento da compreensão do processo saúde-doença;

- a equidade: dimensionada na atenção às necessidades de saúde da população, respeitando-se as diferenças individuais;
- a universalidade: garantia democrática do acesso a essa racionalidade enquanto direito de exercício de cidadania ${ }^{5}$.

Assim, a partir da década de 1980, alguns estados e municípios brasileiros começaram a oferecer atendimento homeopático como especialidade médica aos usuários dos serviços públicos de saúde. Alguns deles criaram quadros de médicos homeopatas e realizaram concursos públicos, porém como iniciativas isoladase, às vezes, descontinuadas, por falta de uma política nacional. Após a criação do SUS, o processo de implantação da homeopatia nos serviços públicos de saúde avançou, e a oferta do atendimento médico homeopático cresceu. Esse avanço pode ser observado no número de consultas em homeopatia que, desde sua inserção como procedimento na tabela do SIA/SUS, vem apresentando crescimento anual em torno de 10\%. Em 1999, foram realizadas e aprovadas 32.254 consultas; já em 2003, foram aprovadas 291.069 consultas médicas em homeopatia ${ }^{6}$. Em 1988, a Comissão Interministerial de Planejamento (Ciplan) - abrangendo os ministérios da Saúde, Educação, Previdência, Trabalho e Planejamento - publicou uma resolução que estabeleceu critérios para a implantação do atendimento homeopático nos serviços públicos de saúde. Iniciou-se, assim, um movimento de expansão dos programas de terapias alternativas, no qual os municípios ganharam autonomia para a execução dos serviços de saúde 7 .

Em maio de 2006, após três anos de amplo debate com a sociedade civil organizada, estados e municípios, além da pactuação pela Comissão Intergestores Tripartite e aprovação pelo Conselho Nacional de Saúde, foi editada a Portaria 971, que aprovou a Política Nacional de Práticas Integrativas e Complementares (PNPIC) e assegura o acesso aos usuários do SUS à medicina tradicional chinesa/acupuntura, homeopatia, fitoterapia e termalismo social. Esta política responde ao anseio de muitos usuários e profissionais de saúde, manifestado nas recomendações de Conferências Nacionais de Saúde desde 1988, além de atender às recomendações da Organização Mundial da Saúde (OMS), principalmente aquela contida no documento "Estratégia da OMS sobre Medicina Tradicional 2002-2005”6. Esta portaria tem ainda como um de seus objetivos o incentivo e apoio a projetos de assistência, ensino e pesquisa homeopáticos nas diversas esferas do SUS, juntamente com outras práticas não convencionais ${ }^{2}$.

Recentemente, em dezembro de 2007, foi publicada a Portaria 3.237, do Ministério da Saúde, que inclui os medicamentos homeopáticos da Farmacopeia Homeopática Brasileira (cerca de 450 medicamentos) para serem disponibilizados aos usuários do SUS, em conformidade com o que recomenda a PNPIC ${ }^{8}$, sugerido por Hahnemann no Organon (p.? $)^{9}$ : 
O Estado, no futuro, depois de compreender a indispensabilidade de medicamentos homeopáticos perfeitamente preparados, fará com que sejam preparados por uma pessoa competente e imparcial a fim de dá-los gratuitamente a médicos homeopatas treinados em hospitais homeopáticos, que tenham sido examinados teórica e praticamente e, assim, legalmente qualificados. O médico pode então se convencer desses instrumentos divinos de curar e também dá-los gratuitamente a seus pacientes, ricos ou pobres.

Assim, é fundamental conhecer as concepções que os estudantes da área da saúde têm sobre a homeopatia, se utilizam ou negligenciam os pressupostos da ciência sistêmica, e observar as fontes de informação a partir das quais obtiveram estes conhecimentos, a fim de caracterizar a homeopatia como objeto de representação social para esse grupo de futuros profissionais.

\section{Representações sociais}

As representações dos acadêmicos de cursos da área da saúde sobre a homeopatia foram abordadas por meio da Teoria das Representações Sociais. O estudo das Representações Sociais surgiu no campo da Psicologia Social, com Serge Moscovici, na década de 1960, como uma abordagem alternativa às teorias tradicionais de cognição social. A representação social se encontra na interface entre o psicológico e o social, e se apresenta como uma atividade de transformação entre os saberes, científico e de senso comum.

O social intervém na construção das representações, de algumas formas: pela influência do contexto de inserção dos indivíduos e grupos, pelos processos comunicativos, pelas experiências prévias que são acumuladas como "bagagens" culturais, pela ideologia, pelos códigos e valores relativos à posição e pertença social. A construção das representações ocorre a partir de experiências, informações, estruturas de pensamento recebidas e transmitidas pela tradição, educação e comunicação social ${ }^{10}$.

A construção das representações sociais das teorias científicas ou de profissões formalizadas - ou seja, as modificações que sofrem à medida que são apropriadas pelos diferentes grupos sociais - deve ser considerada a partir de fatores como posição social, crenças religiosas, políticas e escolaridade. As representações sociais engendram um processo de transformação recíproco de tipos de conhecimento, isto é, do saber científico e do saber de senso comum. As representações sociais são, portanto, uma forma de conhecimento prático, de "pensamento social", que direciona aspectos da comunicação, da compreensão e do domínio do ambiente social, material e ideal ${ }^{10}$.

Assim, as concepções e as representações que os acadêmicos constroem e adotam implicam diretamente as suas práticas e as relações que desenvolverão a partir de sua inserção na atenção básica. Isto oferece aos profissionais de saúde e também a toda a sociedade instrumentos de questionamento e reconstrução de símbolos, ideias e aplicações, interferindo na prática de assistência à saúde.

\section{MÉTODO}

\section{Participantes}

Participaram do estudo acadêmicos de graduação dos cursos de Medicina, Farmácia e Odontologia de uma universidade localizada no Sul do Brasil, de ambos os sexos, que não tiveram a disciplina de homeopatia ministrada. Cor, classe social e estado geral de saúde não foram dados relevantes para esta pesquisa e, sendo assim, não foram considerados.

\section{Caracterização da pesquisa}

A pesquisa é do tipo descritivo-correlacional de base epistemológica qualitativa, pertinente à investigação de fenômenos complexos, como representações, vivências, crenças, valores e significa $\operatorname{dos}^{11,12}$. A pesquisa foi realizada de acordo com as normas da Resolução 196/96 do Conselho Nacional de Saúde, tendo sido aprovada pelo Comitê de Ética em Pesquisa de uma universidade do Sul do Brasil (Coep, Parecer 011/07). Todos os participantes foram esclarecidos sobre os objetivos e a metodologia, mediante leitura e assinatura do Termo de Consentimento Livre e Esclarecido. A pesquisa não apresentou riscos para os participantes, e lhes foi garantido o sigilo, assim como o retorno sobre os resultados.

\section{Instrumento para coleta dos dados}

Os dados foram coletados de julho a setembro de 2007 por meio do preenchimento de um questionário individual, voluntário, aplicado nos primeiros 15 minutos de aula. O questionário foi entregue de forma coletiva em sala de aula, com o auxílio do professor e o consentimento da direção de cada curso. Realizou-se uma análise prévia do questionário a partir de sua aplicação a cinco acadêmicos, que não foram incluídos na pesquisa. As perguntas norteadoras foram as seguintes: (a) O que é homeopatia?; (b) O queé atendimento integral ao usuário do SUS?; (c) Qual a relação da homeopatia com o SUS?; (d) Como é o medicamento homeopático?; (e) Você faz uso da homeopatia?; (f) Como é a consulta homeopática?; (g) Como é o tratamento homeopático?

\section{Procedimentos para análise dos dados}

Os dados coletados foram analisados de acordo com a metodologia da análise de conteúdo categorial temática ${ }^{13}$, que permi- 
tiu identificar a posteriori as categorias de análise em função das generalidades e peculiaridades encontradas nas respostas dos participantes. Procedeu-se a uma análise estatística descritiva, baseada em dados de frequência, percentagem e incidência de ocorrência de respostas ${ }^{14}$.

\section{RESULTADOS E DISCUSSÃO}

A seguir, serão discutidos os resultados das entrevistas, divididos em quatro grupos:

1. perfil dos acadêmicos pesquisados;

2. concepções dos acadêmicos acerca da homeopatia como terapêutica;

3. a homeopatia e o Sistema Único de Saúde (SUS);

4. fontes de informação acerca da homeopatia e do SUS.

\section{Perfil dos acadêmicos pesquisados}

Foram pesquisados 53 alunos dos cursos de Farmácia, Medicina e Odontologia. Destes, 36 (67,9\%) são do sexo feminino e 17 $(32,1 \%)$ do sexo masculino, com predominância do sexo feminino principalmente no curso de Farmácia. A maioria dos acadêmicos tinha entre 19 e 27 anos (77,4\%), destacando-se 5 entrevistados com mais de 40 anos. A grande maioria dos entrevistados era proveniente do município onde está situada a universidade (48\%). Quando perguntados se faziam uso da homeopatia como terapêutica para cuidar da própria saúde, $13,2 \%$ responderam afirmativamente, e dentre estes a maioria era de acadêmicos de Farmácia. Os dados sobre a população estudada estão na Tabela 1.

\section{Concepções dos acadêmicos acerca da homeopatia como terapêutica}

O primeiro objetivo da pesquisa foi levantar informações a respeito das percepções dos estudantes universitários sobre a homeopatia, por meio das perguntas: “O que é homeopatia? O que você sabe sobre homeopatia?". Constatou-se que 36,1\% dos entrevistados atribuíram à homeopatia a representação de que se trata de uma terapia que utiliza produtos naturais (Tabela 2). Resultados semelhantes foram obtidos por Micali et al. ${ }^{15} \mathrm{em}$ estudo que investigou as representações sobre a homeopatia em Vitória (ES) e também constatou a associação da homeopatia a um tratamento natural por $38,4 \%$ da população estudada ${ }^{15}$. Os autores constataram que a representação da homeopatia pelos entrevistados, enquanto tratamento natural, estava relacionada à imagem de um medicamento não elaborado e sem a possibilidade de efeitos colaterais. Esta associação da homeopatia com ervas naturais é bastante comum. Como no estudo realizado recentemente, os usuários entrevistados se referiram à homeopatia como um medicamento natural, mais saudável, sem efeitos colaterais.

Sugerimos aqui que tais respostas estão ligadas ao fato de a homeopatia utilizar plantas para a produção da maioria dos medicamentos homeopáticos. Mas os fundamentos que diferenciam as duas práticas são opostos. A homeopatia se baseia na cura por meio do princípio enunciado por Hipócrates, 'semelhante cura semelhante', com a utilização de uma substância capaz de provocar determinados sintomas num indivíduo sadio e também capaz de curar estes mesmos sintomas apresentados por uma pessoa doente; já a fitoterapia segue a lei do 'contrário cura contrário' $^{1}$. Além desta diferença, também é fundamental que tenham sido empregadas as doses infinitesimais e o processo de dinamização, para então serem considerados medicamentos homeopáticos ${ }^{16}$.

Duas outras concepções, referidas com frequências semelhantes pelos entrevistados, foram que a homeopatia faz uso de medicamentos diluídos e que estes agem por meio da cura pelo semelhante. A referência pelos alunos ao medicamento diluído parece se basear na utilização de doses infinitesimais na preparação dos medicamentos homeopáticos, ou seja, as substâncias ativas são usadas

Tabela 1

Perfil dos acadêmicos pesquisados

\begin{tabular}{|c|c|c|c|c|c|}
\hline \multirow{2}{*}{ Graduação } & \multirow{2}{*}{ Amostra (n) } & $\operatorname{Sexo}(n)$ & \multicolumn{3}{|c|}{ Usuários de homeopatia (n) } \\
\hline & & $\mathrm{M}$ & $\mathrm{F}$ & $\operatorname{Sim}$ & não \\
\hline Farmácia & 25 & 5 & 20 & 5 & 20 \\
\hline Medicina & 18 & 10 & 8 & 1 & 17 \\
\hline Odontologia & 10 & 2 & 8 & 1 & 9 \\
\hline Total & 53 & 17 & 36 & 7 & 46 \\
\hline
\end{tabular}


em doses muito baixas. Historicamente, este é um dos principais questionamentos no meio científico em relação à homeopatia: como substâncias diluídas e agitadas, ou seja, dinamizadas, podem suscitar alguma resposta em sistemas biológicos ou organismos vivos? O modelo farmacológico bioquímico e dose-dependente, ensinado e fundamentado desde o primeiro dia de aula, para estudantes universitários dos cursos de graduação da área da saúde éa referência de prática terapêutica. É, então, a partir desta lógica que emerge o principal alvo das críticas ao modelo homeopático².

A outra resposta também citada pelos entrevistados - a cura pelos semelhantes - foi proveniente, em sua maioria, de alunos do curso de Farmácia (dados não mostrados). Este dado pode es- mento natural e $14 \%$ o efeito placebo ${ }^{17}$. As respostas que associavam a homeopatia a um tratamento alternativo e tratamento único foram mencionadas apenas uma vez para cada uma delas. A frequência das citações e sua relação percentual se encontram na Tabela 2.

Certamente, as respostas fornecidas por estes acadêmicos fornecem elementos bastante relevantes para reflexões, pois espelham a realidade dos cursos de graduação que não trazem a homeopatia em sua grade curricular. Para corrigir conceitos equivocados sobre esta disciplina, seria aconselhável ensinar os aspectos fundamentais desta terapêutica, de forma breve e obri-

Tabela 2

Frequência das respostas relativas às representações sociais da homeopatia

\begin{tabular}{|c|c|c|}
\hline \multirow{2}{*}{ Resposta } & \multicolumn{2}{|c|}{ Frequência } \\
\hline & $\mathrm{N}$ & $\%$ \\
\hline Produtos naturais & 22 & 36,1 \\
\hline Cura através do semelhante & 17 & 27,9 \\
\hline Medicamento diluído & 16 & 26,2 \\
\hline Placebo (psicológico) & 4 & 6,6 \\
\hline Tratamento alternativo & 1 & 1,6 \\
\hline Tratamento único & 1 & 1,6 \\
\hline
\end{tabular}

tar relacionado à grade curricular destes acadêmicos, onde está inserida uma disciplina de apresentação das atribuições possíveis do farmacêutico. Desta forma, mesmo sem terem cursado a disciplina de homeopatia, os estudantes foram norteados por um dos pilares da homeopatia para conceituá-la.

Algumas respostas descrevem a homeopatia como uma terapêutica que emprega medicamento placebo (6,6\%). Apesar de utilizada há mais de dois séculos em diversos países, a homeopatia permanece marginalizada perante a racionalidade científica moderna por estar fundamentada em princípios pouco ortodoxos, que desafiam o pensamento linear característico do paradigma da ciência tradicional, que pressupõe objetividade, estabilidade e simplicidade. Embora seja minoria entre os entrevistados, a concepção de que a homeopatia não emprega substância ativa, curando por meio do efeito placebo, faz coro a outra pesquisa entre estudantes de Medicina, na qual 18\% dos entrevistados consideraram como prerrogativa da homeopatia o trata- gatória, nos primeiros anos das faculdades de área da saúde, em disciplinas mais abrangentes, independentemente do aprofundamento ${ }^{17}$.

Desde 1982, o ensino da homeopatia vem sendo debatido, particularmente no que diz respeito à formação do médico. Alguns trabalhos têm mostrado um crescente interesse dos estudantes de Medicina em conhecer e fundamentar conceitos na área de homeopatia e em outras práticas integrativas e complementares, atribuindo este interesse à inserção dessas práticas no SUS e à crescente procura da população por essas terapias ${ }^{18,19}$. Embora seja oficialmente reconhecida como especialidade médica e até mesmo a OMS recomende sua inserção em serviços públicos de saúde, a homeopatia faz parte dos currículos acadêmicos de Medicina, em sua maioria, apenas como disciplina optativa. Algumas poucas faculdades de Medicina a oferecem como obrigatória, e apenas a Universidade do Rio de Janeiro a tem como residência médica e também como disciplina obrigatória ${ }^{20}$. 
Nas faculdades de Farmácia do Brasil, o ensino de farmacotécnica homeopática passou a ser obrigatório a partir de 1952, com a Lei 1.552. De acordo com um levantamento de dados realizado em 2001, metade dos cursos de Farmácia no Brasil oferecia a disciplina de farmacotécnica homeopática em caráter optativo; de 10\% a 12\% dos cursos estavam implantando a disciplina; $5 \%$ a tinham inserido no programa de outra disciplina; e 35\% não a ofereciam. Atualmente, com a reformulação do ensino em praticamente todas as faculdades de Farmácia, por efeito das novas diretrizes curriculares elaboradas pelo Conselho Nacional de Educação, o ensino de homeopatia voltou a ser o foco das atenções ${ }^{21}$.

Já nos cursos de graduação em Odontologia, raras são as instituições que oferecem esta disciplina como optativa. A formação em homeopatia para estes profissionais, na maioria das vezes, ocorre sob a responsabilidade dos cursos de especialização ou de extensão, normalmente vinculados a associações profissionais e institutos homeopáticos, e que se destinam também aos demais profissionais de saúde. Ainda que não seja reconhecida oficialmente como especialidade odontológica, recentemente o Conselho Federal de Odontologia reconheceu e regulamentou o uso, pelo cirurgião-dentista, de práticas integrativas e complementares à saúde bucal, incluindo a homeopatia ${ }^{22,23}$.

\section{A homeopatia e o Sistema Único de Saúde (SUS)}

AOMS tem incentivado o desenvolvimento de projetos homeopáticos que visem incrementar sua disponibilidade junto aos sistemas públicos de saúde mundiais, de forma coadjuvante aos tratamentos clássicos, por ser a homeopatia considerada uma alternativa eficiente e segura ao tratamento das doenças crônicas.
Com o propósito de incentivar e apoiar projetos de assistência, ensino e pesquisa homeopáticos nas diversas esferas doSUS, juntamente com outras práticas não convencionais, o Ministério da Saúde aprovou, em 2006, a Política Nacional de Práticas Integrativas e Complementares no Sistema Único de Saúde².

Segundo esta abordagem, se constituiu como outro objetivo de pesquisa estudar as concepções dos estudantes sobre a relação entre a homeopatia, oSUS e o princípio da integralidade. Neste aspecto, diante da pergunta "o que é atendimento integral ao usuário do SUS?", a maior parte dos alunos pesquisados soube mencionar o conceito clássico da integralidade, vinculando o indivíduo a seu contexto, seu universo (Tabela 3). Mostraram saber correlacionar o atendimento integral ao sujeito e a compreensão de suas necessidades de forma a contemplar uma atenção totalizadora, levando em conta todos os aspectos relacionados ao usuário. As principais respostas dos entrevistados relativas ao atendimento integral ao usuário do SUS encontram-se na Tabela 3.

$\mathrm{O}$ conceito que estabelece que o atendimento integral ao usuário significa atender o paciente em todas as suas necessidades, nos aspectos biopsicossocial e como um todo, pareceu ser um conceito bem sedimentado entre os acadêmicos. $\mathrm{O}$ fato de $44 \%$ do total de respostas contemplarem estes conceitos nos levou a confrontar a grade curricular e a observar as diferenças entre as respostas dos que tiveram alguma disciplina de saúde coletiva ministrada e os que não tiveram. Os dados demonstram que os alunos que ainda não haviam tido a disciplina de Saúde Pública em sua grade curricular foram os mesmos que não souberam responder a esta pergunta (25,4\%). Também ocorreu que, mesmo tendo tido a disciplina, alguns alunos relacionaram o atendimento integral como sendo aquele em que o paciente deve

Tabela 3

Frequência das respostas relacionadas ao atendimento integral ao usuário do SUS

\begin{tabular}{lcc}
\hline & & Frequência \\
\cline { 2 - 3 } Resposta & $\mathrm{N}$ & $\%$ \\
\hline Atendimento em todas as necessidades do paciente & 15 & 25,4 \\
Atendimento completo custeado pelo SUS & 10 & 16,9 \\
Acesso a todas as especialidades & 8 & 13,6 \\
Paciente como um todo & 7 & 11,9 \\
Biopsicossocial & 4 & 6,8 \\
Não sabe & 15 & 25,4 \\
\hline
\end{tabular}


ter a sua disposição todas as especialidades e todos os serviços custeados pelo SUS.

Além disso, deve ser considerado que, mesmo não tendo interesse em trabalhar nesta especialidade ou área, o aluno deveria ter acesso ao conhecimento para poder optar e entender a prática de outras racionalidades. Assim, pode-se afirmar que a principal contribuição da homeopatia no SUS, sua inserção na grade curricular e sua relação com a integralidade como princípio do SUS se referem ao restabelecimento de uma compreensão mais ampla e global sobre os problemas de saúde, implicando a afirmação de que o desequilíbrio no estado do indivíduo não é exclusivamente orgânico.

Ao serem questionados sobre a relação entre a homeopatia e o SUS, $86,8 \%$ dos alunos referiram desconhecer a relação e 13,2\% afirmaram que o SUS utiliza apenas a alopatia como terapêutica. Destas respostas, podemos sugerir que: primeiro, os alunos não tinham conhecimento da Portaria 971, em vigor desde maio de 2006, e da recém-publicada Portaria 3.237, de dezembro de 2007, que trata da inclusão da homeopatia e de medicamentos homeopáticos no SUS; segundo, desconhecem a realidade das práticas do sistema de saúde de municípios e estados. Reconhecida desde 1980 como especialidade médica pelo Conselho Regional de Medicina, a homeopatia vem sendo praticada por médicos dentro dos consultórios das unidades locais de saúde e de hospitais públicos como iniciativas pessoais destes profissionais, chegando a mais de 290 mil consultas médicas aprovadas em $2003^{6}$. O Brasil tem cerca de 5.500 municípios, mas apenas 157 deles oferecem a terapêutica homeopática em unidades de saúde. Existe, então, um grande passo a ser dado em número de atendimentos em homeopatia, sendo necessário um maior número de médicos homeopatas que atuem no SUS 24

É preciso mencionar que o acesso à informação sobre as diversas modalidades terapêuticas pelos estudantes permitiria uma escolha mais lúcida e possibilitaria ampliar a atuação desses futuros profissionais. Além disso, a inserção da homeopatia como prática oficial contribuiria para promover o envolvimento responsável de usuários, gestores e trabalhadores, e para racionalizar as ações de saúde ${ }^{6,25,26}$. O aumento da demanda de atenção médica em decorrência de problemas de saúde abrangentes, que incluem aspectos psicossociais, desequilíbrio da demanda-oferta dos serviços públicos de saúde e baixa resolubilidade do modelo biomédico, torna necessário redefinir práticas de atenção e reorganização dos serviços ${ }^{27,28}$. E, principalmente, requer reformulações na educação em homeopatia para que estes futuros profissionais possam compreender o papel dessa terapêutica no contexto da saúde pública.
As instituições de ensino superior vêm sendo pressionadas por mudanças no processo de formação e na maneira como a universidade se relaciona com a sociedade. Assim, é fundamental um compromisso com o eixo da integralidade na mudança da graduação em saúde, principalmente daqueles que estão nas interfaces da formação: docentes, estudantes, gestores de educação e gestores do SUS ${ }^{29}$. Embora a homeopatia seja uma racionalidade médica coerente e busque atender as demandas de saúde de forma abrangente e universal, reforçando os princípios norteadores do SUS, é imprescindível seu reconhecimento, valorização e disponibilidade a toda a população, para que possa contribuir na resolução eficiente dos problemas de saúde e na promoção da integralidade da assistência ${ }^{27,28}$.

\section{Fontes de informação acerca da homeopatia e do SUS}

As fontes de informação citadas pelos entrevistados acerca do conhecimento sobre a homeopatia, sua relação com o SUS e a integralidade na atenção são de três tipos:

- Formação acadêmica: informações obtidas por meio de relações formais, de contato direto durante estágios extracurriculares ou nas disciplinas cursadas;

- Experiência pessoal: informações obtidas por meio de relações de amizade ou parentesco, com contato direto com médicos homeopatas e como usuário do SUS;

- Meios de comunicação: informações obtidas por acesso a materiais impressos (artigos científicos, revistas, livros) e internet.

\section{CONSIDERAÇÕES FINAIS}

Humanizar o atendimento aos pacientes, adotar práticas preventivas para o enfrentamento das doenças, construir um viver saudável, recuperando o entendimento do conceito saúde-doença, são os princípios que orientam as políticas do SUS e que vêm ao encontro dos fundamentos da homeopatia. Além disso, a inclusão desta no SUS possibilita ampliar o universo de usuários, configurando o direito de escolha do cidadão.

Em nosso trabalho, verificamos desconhecimento da incorporação da homeopatia no SUS pelos entrevistados e também um nível superficial de informação sobre os pressupostos homeopáticos entre os acadêmicos. Tem-se aqui, portanto, uma lacuna a ser preenchida: a ausência da homeopatia nos currículos e a falta de profissionais que possam ser incorporados pelo SUS, como consequência.

Constatamos que, embora a teoria tenha fornecido alguns subsídios para a elaboração do conceito de integralidade entre os alunos, algumas dúvidas poderiam ser dissipadas na prática 
profissional, com a inserção do estudante no contexto das atividades que estimulem a exploração de conteúdos a partir de situações-problema. É impossível pensar em saúde sem pensar em educação no sentido mais amplo do conhecimento, em sua epistemologia, ou seja, na construção de suas implicações.

A homeopatia é uma terapêutica de ação generalista, que atua em todas as faixas etárias e requer tecnologia simples. A experiência de implantação e consolidação da homeopatia como opção terapêutica nos serviços públicos de saúde pode oferecer informações importantes para subsidiar a organização e integração mais efetiva dessa terapêutica em outros serviços de saúde pública. Isto porque sua integração às demais ações desenvolvidas pelo SUS, juntamente com a ampliação do acesso, vem reforçar os princípios de universalidade, integralidade e equidade.

\section{REFERÊNCIAS}

1. Dantas F. O que é homeopatia? São Paulo: Brasiliense; 1998.

2. Teixeira MZ. Homeopatia: ciência, filosofia e arte de curar. Rev Med (São Paulo). 2006;85(2):30-43.

3. Corrêa AD, Siqueira-Batista R, Quintas LEM, Siqueira-Batista R. Similia Similibus Curentur: revisitando aspectos históricos da homeopatia nove anos depois. Hist Cienc Saude Manguinhos. 2006;13(1):13-31.

4. Brasil. Ministério da Saúde. Secretaria de Políticas de Saúde. Departamento de Atenção Básica. Manual para a Organização da Atenção Básica. Brasília: MS; 2001.

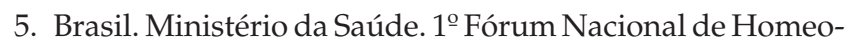
patia: a homeopatia que queremos implantar no SUS: relatório. Brasília: Ministério da Saúde; 2004.

6. Brasil. Ministério da Saúde. Gabinete do Ministro. Portaria no 971, de 3 maio de 2006. Aprova a Política Nacional de Práticas Integrativas e Complementares (PNPIC) no Sistema Único de Saúde. Diário Oficial da União. Brasília, 4 maio 2006; Seção 1, p. 20.

7. Justo CMP, Gomes MHA. A cidade de Santos no roteiro de expansão da homeopatia nos serviços públicos de saúde no Brasil. Hist Cienc Saude Manguinhos. 2007;14(4):1159-1171.

8. Brasil. Ministério da Saúde. Gabinete do Ministro. Portaria no 3.237, 24 de dezembro de 2007. Aprova as normas de execução e de financiamento da assistência farmacêutica na atenção básica em saúde. Diário Oficial da União. Brasília, 26 dez. 2007; Seção 1, p. 16.
9. Hahnemann S. Organon da Arte de Curar. $6^{a}$ ed. São Paulo: Robe; 1996.

10. Jodelet D. Representation Sociale. In: Grand Dictionnaire de la Psychologie. Paris: Larousse; 1991.

11. Bauer MW, Aarts B. A construção do corpus: um princípio para a coleta de dados qualitativos. In: Bauer MW, Gaskell $\mathrm{G}$, orgs. Pesquisa qualitativa com texto, imagem e som. Petrópolis: Vozes; 2002. p. 39-63.

12. Minayo MC, Sanches O. Quantitativo-qualitativo: oposição ou complementaridade? Cad Saúde Pública. 1993;9(3):239-62.

13. Bardin L. Análise de Conteúdo. 3ª̣ ed. Lisboa: Edições 70; 2004.

14. Barbetta PA. Estatística Aplicada às Ciências Sociais. Florianópolis: UFSC; 1994.

15. Micali IA, Salume S, Machado VLT. Imagens da homeopatia na comunidade de Vitória-ES. Rev Homeopatia. 1995;60:27-33.

16. Fontes OL. Medicamento Homeopático. In: Farmácia Homeopática: teoria e prática. 2ª ed. São Paulo: Manole; 2005.

17. Teixeira MZ. Homeopatia: desinformação e preconceito no ensino médico. Rev Bras Educ Med. 2007;31(1):15-20.

18. Teixeira MZ, Chin AL, Martins MA. Homeopathy and acupuncture teaching at Faculdade de Medicina da Universidade de São Paulo: the undergraduates attitudes. Sao Paulo Med J. 2005; 123(2):77-82.

19. Külkamp IC, Burin GD, Souza MHM, Silva P, Piovezan AP. Aceitação de práticas não-convencionais em saúde por estudantes de medicina da Universidade do Sul de Santa Catarina. Rev Bras Educ Med. 2007;31(3):229-35.

20. Salles SAC. A presença da homeopatia nas faculdades de medicina brasileiras: resultados de uma investigação exploratória. Rev Bras Educ Med. 2008;32(3):283-90.

21. Corrêa AD, Leite SQM. Ensino da homeopatia na graduação em farmácia: concepções e práticas pedagógicas em instituições do estado do Rio de Janeiro. Interface Comun Saúde Educ. 2008;12:267-80.

22. Durgante LP. Expectativas dos indivíduos homeopatas de Itajaí a respeito de sua participação no SUS local. Florianópolis; 2006. Mestrado [Dissertação] - Universidade Federal de Santa Catarina.

23. Conselho Federal de Odontologia. Resolução no. 82, de 22 de setembro de ,2008. Reconhece e regulamenta o uso pelo cirurgião-dentista de práticas integrativas e complementares à saúde bucal. [online]. [acesso em 29 dez. 2008]. Dispo- 
nível em: http://www.cfo.org.br/download/pdf/forum_praticas_integrativas/resolucao_82_2008.pdf

24. Gutierrez M. Homeopatia no SUS. Pharm Brasileira. 2008;63:9-16.

25. Luz MT. A arte de curar versus a ciência da doença: história social da homeopatia no Brasil. São Paulo: Dynamis; 1996.

26. Barbosa MA, Fonseca APM, Bachion MM, Souza JT, Faria RM, Oliveira LMAC, Andraus LMS. Terapias alternativas de saúde $x$ alopatia: tendências entre acadêmicos de medicina. Rev Eletrônica Enferm [periódico na Internet]. 2001[acesso em 30 mar. 2008];3(2): [aproximadamente 9p.]. Disponível em: http://www.fen.ufg.br/revista/index.htm

27. Santanna C, Hennington EA, Junges JR. Prática médica homeopática e a integralidade. Interface Comun Saúde Educ. 2008;12(25):233-46.

28. Dantas F. Homeopatia e Atenção à Saúde em Serviços Públicos. Cult Homeopat. 2007; 1813-5.

29. Ceccim RB, Feuerwerker LCM. Mudança na graduação das profissões de saúde sob o eixo da integralidade. Cad Saúde Pública. 2004;20(5):1400-10.

\section{CONTRIBUIÇÃO DOS AUTORES}

Gecioni Loch-Neckel e Françoise Carmignan foram responsáveis pelo planejamento do estudo, aplicação dos questionários, análise e discussão dos dados, e redação do artigo. Maria Aparecida Crepaldi conduziu a elaboração do estudo e contribuiu na revisão final do artigo.

\section{CONFLITO DE INTERESSES}

Declarou não haver.

\section{ENDEREÇO PARA CORRESPONDÊNCIA}

Gecioni Loch-Neckel

Servidão Fidélis Govoni, 75

Morro das Pedras - Florianópolis

CEP. 88066-050 SC

E-mail: gneckel@hotmail.com 